\title{
INTRINSIC CAPACITY AS A DETERMINANT OF PHYSICAL RESILIENCE IN OLDER ADULTS
}

\author{
J.K. CHHETRI ${ }^{1,2,3}$,Q.-L. XUE ${ }^{3,4}$, L. MA ${ }^{1,2}$, P. CHAN ${ }^{1,2}$, R. VARADHAN ${ }^{4,5}$
}

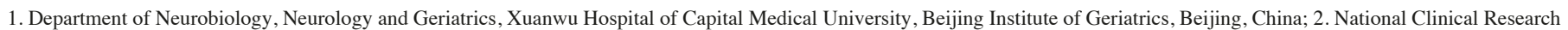

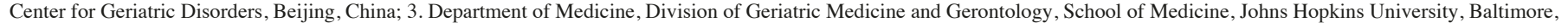
MD, USA; 4. Center on Aging and Health, Johns Hopkins Medical Institutions; USA; 5. Division of Biostatistics and Bioinformatics, Sidney Kimmel Comprehensive Cancer Center,

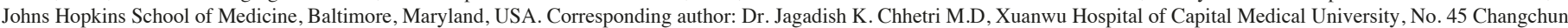

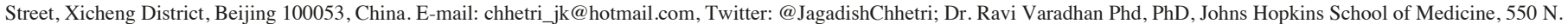
Broadway, Suite 1103-A, Baltimore, MD 21205, USA,E-mail: ravi.varadhan@jhu.edu, Twitter: @Tyche63
\end{abstract}

\begin{abstract}
The traditional disease-oriented model of healthcare is inadequate to address the needs of the older population. Greater attention should be given to strategies that promote healthy aging. Recently proposed constructs of intrinsic capacity (IC) and physical resilience (PR) hold great potential to reshape geriatric medicine and aging research. These constructs accentuate the positive health attributes of older people in contrast to the popular frailty construct that is centered on functional deficits. IC was introduced by the World Health Organization (WHO) as a composite of all the physical and mental capacities. WHO has emphasized enhancement of IC throughout the life course so as to maintain functional ability in old age. PR, recently highlighted by the National Institute on Aging, is the ability to successfully cope with stressors. High levels of resilience can result in desirable clinical and functional outcomes after stressors. Therefore, it is important to understand the underlying physiology of PR and the risk factors contributing to diminished PR. The main goal of this article is to explore the potential relationship between IC and PR. Based on a classical theory of aging, we postulate that IC is a determinant of PR and is also a high-level integrative measure of physiologic reserve which is the fundamental factor underlying one's ability to withstand stressors. A major implication of our postulates is that even though IC is only one of the many determinants of PR, it could serve as an important intervenable target for enhancing resilience in older adults.
\end{abstract}

Key words: Resilience, physiologic reserve, integrated care, aging, frailty.

\section{Introduction}

Population aging is evident globally. The traditional diseaseoriented model of healthcare has proven to be inadequate to address the needs of burgeoning older population. Consequently, there has been an increased interest in aging research and geriatrics to identify strategies that promote healthy aging. Recently proposed constructs of intrinsic capacity (IC) and physical resilience (PR) hold great potential to reshape the future of geriatric medicine and aging research. These constructs accentuate the positive health attributes of older people in contrast to the popular frailty construct that is centered on functional deficits. This is a conceptual shift from approaches that focused on the prevention of adverse health states such as frailty and disability to a new paradigm that focuses on promoting positive attributes of health.

IC is defined as an aggregate summary of the physical and mental capacities of an individual (1). It is a relatively new concept proposed by the World Health Organization (WHO) in 2015 in order to capture the health trajectory of an older adult from a functional perspective rather than the traditional diseaseoriented perspective. WHO has launched an innovative care plan known as the Integrated Care for Older People (ICOPE) (2) approach which largely centers around the concept of IC. This approach advocates that the key to strengthening healthy aging is by optimizing IC and maintaining functional ability at an individual level.
$\mathrm{PR}$ is another new concept in geriatrics promoted by the National Institute on Aging (NIA) (3). Broadly speaking, it is the ability to successfully cope with stressors. PR has been defined as one's ability to resist decline or recover from functional decline after a major health stressor $(4,5)$. Another definition characterizes PR as the ability of a system to recover from a perturbation of a sufficiently large magnitude (a stressor) that the system is pushed into a state far from its original equilibrium state, ultimately retaining essential identity and function (6). A better understanding of PR could enable identification of underlying mechanisms of recovery and generate potential targets (both from clinical and public health perspectives) for preventing functional decline in old age.

It is of particular significance that both IC and PR emphasize the positive health attributes of older adults in contrast to the popular concept of frailty which is a clinical syndrome of diminished reserve capacity that increases vulnerability to stressors (7). Nonetheless, both IC and PR overlap with the concept of frailty $(6,8,9)$. In fact, IC may be considered as an evolution of the frailty concept - largely driven by positive aspects of aging, self-empowerment of older individuals (through self-monitoring of functions). IC emphasizes the importance of extrinsic factors such as the environment one lives in (8). We have described distinctions between these concepts in Table 1. IC and PR are new concepts that have not been adequately studied. It is possible that they can be misunderstood and misapplied, as is often the case with 
Table 1

Distinction between Frailty, Intrinsic Capacity and Physical resilience

\begin{tabular}{|c|c|c|c|}
\hline & Frailty & Intrinsic capacity & Physical Resilience \\
\hline Construct & $\begin{array}{l}\text { A clinical syndrome that reflects a state of } \\
\text { increased vulnerability to multiple adverse } \\
\text { outcomes. There is a single state of frailty at any } \\
\text { given time. }\end{array}$ & $\begin{array}{l}\text { Reflects a composite of all mental and physical } \\
\text { capacities (represented by } 5 \text { major physiological } \\
\text { domains) that can be thought of as a high-level } \\
\text { integrative measure of physiologic reserve. Thus, } \\
\text { there is a single global IC value at any given time. }\end{array}$ & $\begin{array}{l}\text { Reflects a composite of adaptive physiological and } \\
\text { molecular processes in the face of physical stressors } \\
\text { that are largely influenced by pre-determinants- i.e., } \\
\text { genetics, environment, reserves. PR is defined only } \\
\text { when the triad of system, state and stressors are } \\
\text { specified. Thus, there is not a single, global PR, but } \\
\text { multiple PRs. }\end{array}$ \\
\hline Trajectory & $\begin{array}{l}\text { Cascade of health deficits and limitations in old } \\
\text { age. Intrinsically, a dynamic construct but measures } \\
\text { are largely static. }\end{array}$ & $\begin{array}{l}\text { Life-long spectrum of positive attributes. } \\
\text { Intrinsically, a static construct that is defined } \\
\text { without reference to any particular stressor or } \\
\text { time, but may change at different time points. }\end{array}$ & $\begin{array}{l}\text { Life-long spectrum of positive attributes but response } \\
\text { entails at certain time points (i.e., post-stressor). } \\
\text { Intrinsically a dynamic construct in the sense of being } \\
\text { a characteristic of the dynamic response to a stressor. }\end{array}$ \\
\hline Outcomes & $\begin{array}{l}\text { Disability and loss of independence as primary } \\
\text { outcomes. }\end{array}$ & Functional abilities as primary outcomes. & Functional recovery as primary outcome. \\
\hline Interventions & Comprehensive geriatric assessment. & $\begin{array}{l}\text { Intervention targeted on improving health care } \\
\text { by integrating services so as to provide a better } \\
\text { environment. }\end{array}$ & $\begin{array}{l}\text { Intervention targeting stress-response mechanisms, as } \\
\text { well as improving the physiologic reserve. }\end{array}$ \\
\hline Potential purpose & $\begin{array}{l}\text { Primarily used for risk stratification, i.e. identify } \\
\text { people at high-risk for adverse outcomes to } \\
\text { invasive procedures. Could serve as an entry point } \\
\text { for a personalized care. }\end{array}$ & $\begin{array}{l}\text { Assist in developing public health strategies to } \\
\text { promote healthy aging. }\end{array}$ & $\begin{array}{l}\text { Assist in clinical decision making and developing } \\
\text { care models (both acute or long term) and identify } \\
\text { preventive strategies to improve resilience and } \\
\text { promote healthy aging such as prehab, Enhanced } \\
\text { recovery after surgery, and rehab. }\end{array}$ \\
\hline
\end{tabular}

emerging constructs. Indeed, the aging field is fragmented with various poorly-delineated concepts and disparate empirical findings. There is a real need for a unifying theory that can advance both gerontological research and geriatric practice. In this regard, the novel concepts of IC and PR hold great promise.

The main objective of this paper is to clarify the relationship between IC and PR. How are they distinct? What is the link between them? To achieve our goals, we lean upon a theory of aging developed in the 1960s by Strehler and Mildvan. We discuss the fundamental notion of physiologic reserve and postulate how it connects IC and PR.

\section{Description of IC and PR}

\section{IC and its operationalization}

IC is defined as a composite of all the physical and mental capacities (including psychological capacities) of an individual, that one can draw at any time point during his/her life period (1). WHO's Integrated Care for Older People (ICOPE) approach suggests that one's functional ability depends on IC, the environment, and the interaction between the two (2). The construct of IC encompasses a holistic approach to one's functional status over time. Thus, longitudinal evaluation of an individual's IC trajectory (without consideration of a specific medical condition) could provide opportunities for early intervention to maintain functional ability even in old age. In fact, an ambitious "INSPIRE" project is set to investigate the trajectories of IC centered pathway for healthy aging (10), which could further elucidate our understanding of the IC concept.

IC is currently operationalized based upon the recently developed ICOPE approach guidelines. IC includes five major domains and is assessed as described below:

1) Locomotion: Chair rise test.

2) Cognition: Working memory, orientation, memory recall.

3) Vitality: Weight loss, recent loss of appetite.

4) Psychology: Feeling down/depressed/hopeless or little interest in doing things.

5) Sensory: Hearing loss, vision loss.

The five domains of IC were chosen based on the International Classification of Functioning, Disability and Health (ICF) framework, which has been described in detail by Cesari et.al. (1). These five domains of IC interact with each other (i.e., interactions across multiple organs and physiological systems) and only the global IC may provide a meaningful measure of an individual's level of functional ability. IC is conceptualized as a composite measure where the component domains are treated as formative indicators (causal indicators) (11). Said differently, IC is causally determined by the five domain-specific indicators, and hence can be estimated as a composite index.

\section{$P R$ and its operationalization}

$\mathrm{PR}$ is an emerging dynamic construct and that is highly relevant in the context of successful aging (4). Broadly speaking, it is the ability to successfully cope with stressors. We argue that PR is unambiguously defined only when we specify the "triad" of \{system, state, and stressor\}. For example, we can say that the whole person is resilient to coronavirus infection in terms of death. Here the system is the whole person; the state is survival status; and the stressor 
is coronavirus infection. While the person may be resilient to coronavirus in terms of death as the state, he or she may not be resilient in terms of another state variable, e.g., muscle strength if he or she becomes greatly weakened as a result. Therefore, we posit that PR is not well defined unless all of the three aspects - system, state, and stressor, are clearly specified. This is an essential conceptual distinction between PR and all the other existing concepts in aging including frailty. For example, frailty is characterized as a state of increased vulnerability to stressors. However, there is no explicit specification of the system(s), state, or stressor. The implication is that frailty is a global state of increased vulnerability to all stressors commonly encountered by a person. A desirable consequence of requiring complete specification of the triad of system, state, and stressor is that it becomes a lot easier to operationalize PR. On the other hand, lack of such specificity has impeded progress in frailty research and translation and has resulted in a proliferation of instruments for frailty assessment.

Our ability to understand why and how (i.e., the underlying mechanism) some individuals retain or regain function despite suffering injury or being severely ill, may assist in identifying individuals who may be at high risk or those who benefit more, long before they encounter a particular stressor. This could further aid in developing intervention strategies that could increase resilience and promote healthy aging.

How do we quantify PR? This is an active area of research. Three potential strategies to quantify PR have- been proposed (4). First, aging phenotypes such as frailty and fatigability can be used to approximate (as potential surrogates) PR. Age discrepancy, i.e. the difference between actual chronologic age and an imputed biologic age could be used to approximate PR cross-sectionally. Both of these approaches assess PR without direct knowledge of the stressor and its response. The third approach of quantifying PR is a direct approach that is based on the trajectories of recovery in response to a stressor. This approach requires assessment of different measures of health at two or more times and requires a well-defined stressor (12).

Varadhan et al. proposed a stimulus-response modeling paradigm to characterize the loss of resilience in frail older adults (5). The approach is based on the theory of dynamical systems, where we perturb the system with a controlled stimulus and measure the characteristics of its response including the degree of stimulation and the rate of recovery. The degree of correspondence of these stimulus-response estimates with real-life PR would primarily depend on the relevance of the chosen physiological system to an actual stressor. However, it should be noted that real-life PR will also be determined by the type and magnitude of the stressor, and other factors such as genetics, environment, and psychosocial factors.

\section{What is the distinction between IC and PR?}

At first glance, IC and PR appear to share some common characteristics, such as they both capture the presence of positive health attributes. Although extensively based upon one's biological reserve, both are influenced by external factors or the environment. Both are driven by the model of function-centered personalized care rather than the traditional disease-centered models ("one size fits all" type of care model). Nevertheless, these novel entities are distinct in various aspects which we have outlined in Table 1. Investigators might want to keep these distinctions in mind while implementing these constructs.

\section{How are IC and PR Related?}

PR is the capacity to change in order to maintain the same identity i.e., "bend rather than break". In the presence of a stressor, one's functional outcomes could largely depend on the nature of the stressor, and the physiological and molecular processes governing the homeostasis of the stress-response systems. However, PR can also be influenced by pre-existing determinants, such as age, comorbid conditions, molecular changes, various psychosocial factors, or the environment. These pre-existing determinants could provide a threshold for any individuals or organism's resilience (even in the absence of a particular stressor). A recent study by Gijzel and colleagues has highlighted the importance of measurement of factors (referred to as dynamic indicators of resilience) such as physical activity, heart rate, mental well-being in predicting recovery following hospitalization of older patients (13). Furthermore, past studies have shown age, mental status, mobility, nutritional status, and comorbidity to be major determinants of regaining activities of daily livings (ADL) after hospital admissions $(14,15)$. Handgrip strength which is also a strong measure of vitality has been shown to represent higher IC (16) and to predict functional recovery in multiple scenarios $(13,17)$. The importance of psychosocial factors in determining recovery after an insult has been well established in psychological resilience studies and various scales have been validated (18).

The five domains of IC, locomotion, vitality, cognition, psychology, and sensory capacity represent an individual's level of global (i.e., whole system) functionality. As described above, these domains (excluding sensory) have been shown to determine one's functional recovery either together or separately following exposure to health adversity. Sensory capacity captured through hearing and vision is a major channel connecting individuals to their environment (which is known to influence one's PR). Undoubtedly, individuals with better sensory capacity tend to exhibit a higher level of independence (19) and enhanced psychosocial well-being. These domains of IC while used as a summary score showed a direct relationship with loss of function defined by ADL and Instrumental activities of daily livings (IADL) in a longitudinal 
Figure 1

Concept model showing the link between intrinsic capacity, physiologic reserve and physical resilience

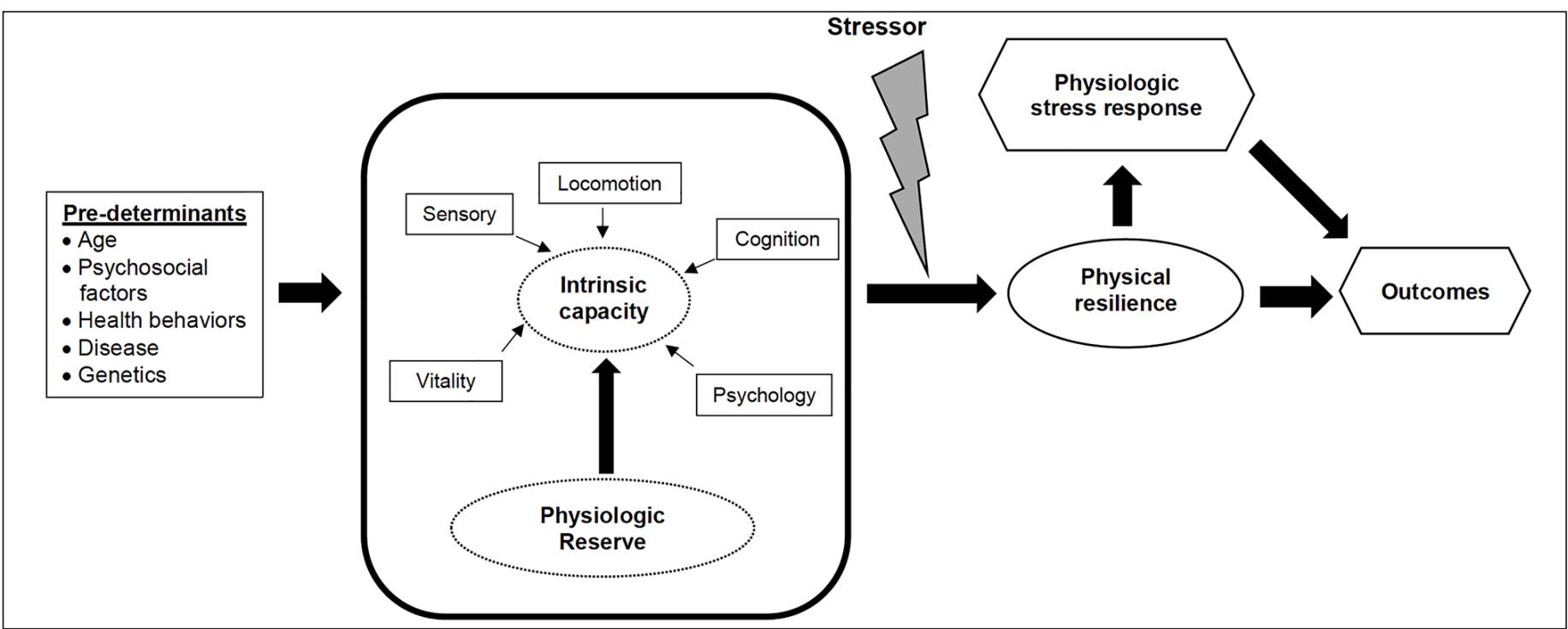

Physical resilience (PR) is a dynamical concept that is defined only under conditions when an organism is exposed to a stressor. The physiological response to the stressor is governed by PR. Long-term outcomes such as physical and cognitive functions, and clinical outcomes will be better for organisms with larger PR. Figure 1 also shows the pre-determinants of PR in-

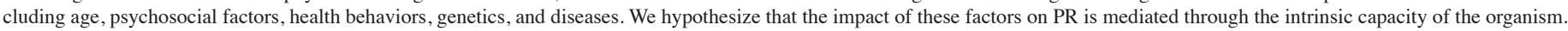
Intrinsic capacity is a high-level integrative measure of the underlying physiologic reserve of the organism, which is the weighted sum of organ-specific reserves (also see Figure 2).

cohort of older community-dwelling older adults (20). The authors have also shown that the risk factors of aging such as age, sex, wealth, education, and multimorbidity (which are potential determinants of PR) were mediated through IC (20). Besides, not only functional limitation but other negative outcomes including falls and mortality (possible trajectory for poor resilience) have been shown to be associated with poor IC in nursing home residents (21).

Thus, IC appears to be a strong determinant of functional recovery. Therefore, we hypothesize that IC is a major determinant of PR (Figure 1). Our hypothesis could be strengthened by utilizing the concept of physiologic reserve. Physiologic reserve could provide a better insight into the higher level dynamic relation between IC and PR. One's level of physiologic reserve could determine his/her intrinsic capacity. Alternatively, IC can also be viewed as a high-level representation (or manifestation) of underlying physiologic reserve (we discuss the rationale for this in the following section), which was previously noted by Whitson et. al (9). Physiologic reserve could also play a key role in determining functional recovery. For instance, in a recent study, ColonEmeric et.al. investigated resiliency following hip fracture in older adults, self-reported pre-fracture functions (described as pre-stressors status) were the most predictive of high resiliency (22). Interestingly, what Colon-Emeric et al. denoted as prestressors status measures largely resemble the domains of IC, and the highly resilient groups are most likely to be individuals with higher levels of IC. Indeed, the dynamics of functional recovery also depends on other factors that we have highlighted in Figure 1 such as age, psychosocial factors, genetics, disease, and other health behaviors.
Figure 2

Physiologic reserve as the link between intrinsic capacity and physical resilience (based on the Strehler and Mildvan theory of aging)

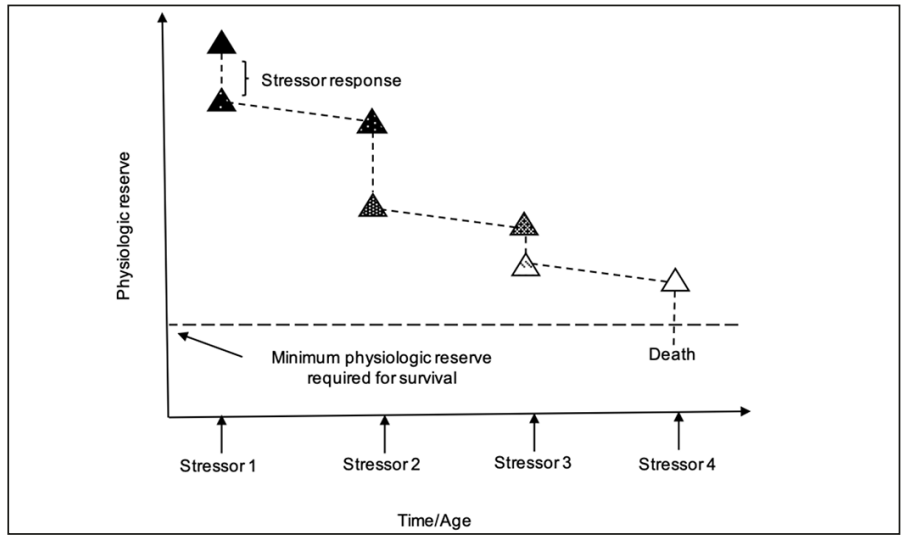

Darker triangle denotes a robust system, which has a higher physiologic reserve and its integrative function or Intrinsic Capacity (IC), therefore, with higher level of physical resilience(PR). Every time a system is exposed to a stressor it uses some of its physiologic reserve (depending on the magnitude of stressor) to overcome the challenge (that leads to a reduction in IC), therefore will have lower level of PR. Gradual exposure to multiple stressors over time reduces the physiologic reserve/IC/PR greatly leaving the system vulnerable (triangle gets lighter in the figure), ultimately leading to death.

\section{Physiologic Reserve as the link between IC and PR}

Strehler and Mildvan (hereafter referred to as "SM") proposed a theory of aging where they considered an organism that is exposed to stressors during its life course (23). The organism consists of several physiological subsystems each of which has a certain maximum ability to restore its 


\section{INTRINSIC CAPACITY AND PHYSICAL RESILIENCE}

original conditions after a stressor. SM defined "vitality" as the capacity of the organism to survive, as quantified by a weighted average of the maximum work capacity minus the basal work output (basal work output is the work required to maintain homeostasis under minimal stress conditions) of each physiological subsystem involved in stress response, which in fact is its physiological reserve. SM postulated that physiologic reserve declined linearly with age. An organism has a certain peak physiologic reserve at some age (typically, maximum physiologic reserve is attained between 25-30 years of agedue to overall system development); thereafter its physiologic reserve declines at a linear rate (due to aging). Nathan Shock studied the physiological processes of various organ systems across different ages between 30 to 70 years and found that their capacities generally declined at a linear rate (24). Sehl and Yates quantified the rate of declines of 13 organ systems (e.g., musculoskeletal, endocrine, thermoregulatory, immunological, etc.) and some integrative functions that span multiple organ systems between the ages of 30 and 70 years (25). They found that the declines are generally around $0.5-2$ percent per year. Thus, we can assume that the physiologic reserve, which is an aggregate of component reserve capacities, also declines at a similar linear rate. The decrease in physiologic reserve with aging should correlate quite well with the decline in IC (i.e., a composite of integrative functions of various systems), i.e. the rate of decline of integrative functions involved in IC is likely similar to the typical rates of individual organ systems. For instance, Sehl and Yates reported that maximum oxygen consumption and exercise tolerance had declines of almost one percent per year (25). Thus, IC can be viewed either as a surrogate measure of physiologic reserve or as a high-level integrative measure of the underlying physiologic reserve (Figure 1).

Based on the SM theory, it is easily seen that physiologic reserve is a fundamental determinant of resilience to stressors. The organism is exposed to stressors of different magnitudes during its life course. Each exposure to stressor demands a certain amount of energy for recovery. As per the SM theory, declines in physiologic reserve with age results in decreased energy available to adequately respond to stressors. If the energy demand exceeds the physiologic reserve of the organism, the organism would die. It survives if the energy demand for responding to the stressor does not exceed its physiologic reserve at that age. Assuming an exponential distribution for the magnitude of stressors, SM showed that their model yielded the well-known, empirically observed the Gompertz law of mortality. Thus, the concept of physiologic reserve provides a plausible link between IC and PR (Figures 1 and 2).

\section{Other Surrogate Measures of Physiologic Reserve}

In the previous section, we proposed IC as a surrogate of physiologic reserve that can also be viewed as a high- level integrative measure of PR. Alternative surrogates of physiologic reserve can be constructed in the context of evaluating resilience to clinical stressors. Here, by surrogate measures, we mean any high-level integrative measure that captures the same level of physiologic reserve as IC. For example, measures such as VO2 max (maximum oxygen consumption), gait speed, or grip strength that are long known to represent one's level of functioning may be suitable candidates (26-28). Frailty phenotype can also serve as a surrogate of physiologic reserve. A self-reported health questionnaire such as the 36 items short-form survey (SF-36) that reflects an individual's overall functional status could be a measure to capture a person's physiologic reserve equally as IC. In the study by Colon-Emeric et al (22), the authors have proposed that a few pre-stressor measures such as grip strength, gait speed, self-reported activity, or IADL were sufficient to define resiliency groups (slope of recovery trajectory were parallel in all groups) after hip fracture.

Are these alternative measures better surrogates of physiologic reserve than IC? Are they better predictors of resilience to stressors than IC? Do any of these measures show responsiveness to intervention, making them suitable targets for enhancing the physical resilience of older adults? These questions need to be evaluated in future studies.

\section{Summary}

Based on a classical theory of aging and the concept of the physiologic reserve, we postulate a link between IC and PR. We have proposed that IC can be viewed as a highlevel integrated measure of physiologic reserve and hence, it can serve as a determinant of PR in older adults. A major implication of our proposal is that even though IC is only one of the many determinants of PR, it could provide an important intervenable target for enhancing resilience in older adults. We hope to spur discussion, debate, and even testing of the ideas proposed here about the relationships between intrinsic capacity, physiologic reserve, and physical resilience.

Acknowledgement: PC is supported by the National Key R\&D Program of China, [grant no. 2018YFC1312001]; Beijing Municipal Commission on Science and Technology [grant no. D07050701130000 and 07050701130701]. QLX is supported in part by the National Institute on Aging at the National Institutes of Health [grant no. P30AG021334]. RV is supported by the National Institute on Aging [grants UH2AG056933 and UH3AG056933].

Declaration of Competing Interests: All authors declare no conflicts of interest.

Author Contributions: JC and RV responsible for drafting the manuscript, all other authors contributed substantially in conceptualization, critical revision, and preparation of the manuscript.

\section{References}

1. Cesari M, Araujo de Carvalho I, Amuthavalli Thiyagarajan J, Cooper C, Martin FC, Reginster J-Y, Vellas B, Beard JR. Evidence for the Domains Supporting the Construct of Intrinsic Capacity. J Gerontol A Biol Sci Med Sci 2018;73:1653-1660.

2. WHO । WHO Guidelines on Integrated Care for Older People (ICOPE) WHO Available at: http://www.who.int/ageing/publications/guidelines-icope/en/. Accessed January 10,2020 .

3. Hadley EC, Kuchel GA, Newman AB, Workshop Speakers and Participants. Report: 


\section{THE JOURNAL OF NUTRITION, HEALTH \& AGING}

NIA Workshop on Measures of Physiologic Resiliencies in Human Aging. J Gerontol A Biol Sci Med Sci 2017;72:980-990.

4. Whitson HE, Duan-Porter W, Schmader KE, Morey MC, Cohen HJ, Colón-Emeric CS. Physical Resilience in Older Adults: Systematic Review and Development of an Emerging Construct. J Gerontol A Biol Sci Med Sci 2016;71:489-495.

5. Varadhan R, Seplaki CL, Xue QL, Bandeen-Roche K, Fried LP. Stimulus-response paradigm for characterizing the loss of resilience in homeostatic regulation associated with frailty. Mech Ageing Dev 2008;129:666-670.

6. Varadhan R, Walston JD, Bandeen-Roche K. Can a Link Be Found Between Physical Resilience and Frailty in Older Adults by Studying Dynamical Systems? J Am Geriatr Soc 2018;66:1455-1458.

7. Fried LP, Tangen CM, Walston J, Newman AB, Hirsch C, Gottdiener J, Seeman T, Tracy R, Kop WJ, Burke G, McBurnie MA, Cardiovascular Health Study Collaborative Research Group. Frailty in older adults: evidence for a phenotype. J Gerontol A Biol Sci Med Sci 2001.56:M146-156.

8. Belloni G, Cesari M. Frailty and Intrinsic Capacity: Two Distinct but Related Constructs. Front Med (Lausanne) 2019;6:133.

9. Whitson HE, Cohen HJ, Schmader KE, Morey MC, Kuchel G, Colon-Emeric CS Physical Resilience: Not Simply the Opposite of Frailty. Journal of the American Geriatrics Society 2018;66:1459-1461.

10. P. De Souto Barreto, Guyonnet S, I. Ader, et al. 2020. The INSPIRE research initiative: a program for GeroScience and healthy aging research going from animal models to humans and the healthcare system. doi:10.14283/JFA.2020.18.

11. Bollen K, Lennox R. Conventional Wisdom on Measurement: A Structural Equation Perspective. Psychological Bulletin 1991;110:305-314.

12. Colón-Emeric C, Pieper CF, Schmader KE, Sloane R, Bloom A, McClain M Magaziner J, Huffman KM, Orwig D, Crabtree DM, Whitson HE. Two Approaches to Classifying and Quantifying Physical Resilience in Longitudinal Data. J Gerontol A Biol Sci Med Sci, 2019. doi:10.1093/gerona/glz097.

13. Gijzel SMW, Rector J, van Meulen FB, van der Loeff RS, van de Leemput IA, Scheffer M, Olde Rikkert MGM, Melis RJF. Measurement of Dynamical Resilience Indicators Improves the Prediction of Recovery Following Hospitalization in Older Adults. J Am Med Dir Assoc, 2019. doi:10.1016/j.jamda.2019.10.011.

14. Gill TM, Robison JT, Tinetti ME. Predictors of Recovery in Activities of Daily Living Among Disabled Older Persons Living in the Community. J Gen Intern Med 1997;12:757-762.

15. Boyd CM, Landefeld CS, Counsell SR, Palmer RM, Fortinsky RH, Kresevic D, Burant C, Covinsky KE. Recovery of activities of daily living in older adults after hospitalization for acute medical illness. J Am Geriatr Soc 2008;56:2171-2179.
16. Ramírez-Vélez R, Correa-Bautista JE, García-Hermoso A, Cano CA, Izquierdo M. Reference values for handgrip strength and their association with intrinsic capacity domains among older adults. J Cachexia Sarcopenia Muscle 2019;10:278-286.

17. Medina-Mirapeix F, Bernabeu-Mora R, Sánchez-Martínez MP, Gacto-Sánchez M, Martín San Agustín R, Montilla-Herrador J. Patterns and Predictors of Recovery from Poor Health Status Measured with the Chronic Obstructive Pulmonary Disease (COPD) Assessment Test in Patients with Stable COPD: A Longitudinal Study. Journal of Clinical Medicine 2019;8:946.

18. Gooding PA, Hurst A, Johnson J, Tarrier N. Psychological resilience in young and older adults. Int J Geriatr Psychiatry 2012;27:262-270.

19. At J, Bryce R, Prina M, Acosta D, Ferri CP, Guerra M, Huang Y, Rodriguez JJL, Salas A, Sosa AL, Williams JD, Dewey ME, Acosta I, Liu Z, Beard J, Prince M. Frailty and the prediction of dependence and mortality in low- and middle-income countries: a 10/66 population-based cohort study. BMC Med 2015;13:138.

20. Beard JR, Jotheeswaran AT, Cesari M, Araujo de Carvalho I. The structure and predictive value of intrinsic capacity in a longitudinal study of ageing. BMJ Open 2019;9:e26119.

21. Charles A, Buckinx F, Locquet M, Reginster J-Y, Petermans J, Gruslin B, Bruyère O. Prediction of adverse outcomes in nursing home residents according to intrinsic capacity proposed by the World Health Organization. J Gerontol A Biol Sci Med Sci doi:10.1093/gerona/glz218.

22. Colón-Emeric C, Whitson HE, Pieper CF, Sloane R, Orwig D, Huffman KM, Bettger JP, Parker D, Crabtree DM, Gruber-Baldini A, Magaziner J. Resiliency Groups Following Hip Fracture in Older Adults. J Am Geriatr Soc, 2019. doi:10.1111/ jgs.16152.

23. Strehler BL, Mildvan AS. General Theory of Mortality and Aging. Science 1960;132:14-21

24. Shock NW. Age changes in some physiologic processes. Geriatrics 1957;12:40-48.

25. Sehl ME, Yates FE. Kinetics of human aging: I. Rates of senescence between ages 30 and 70 years in healthy people. J Gerontol A Biol Sci Med Sci 2001.56:B198-208.

26. Rantanen T, Guralnik JM, Foley D, Masaki K, Leveille S, Curb JD, White L. Midlife Hand Grip Strength as a Predictor of Old Age Disability. JAMA 1999;281:558-560.

27. Hirsch $\mathrm{CH}$, Bůžková P, Robbins JA, Patel KV, Newman AB. Predicting late-life disability and death by the rate of decline in physical performance measures. Age Ageing 2012;41:155-161.

28. Weibel ER, Taylor CR, Hoppeler H. The concept of symmorphosis: a testable hypothesis of structure-function relationship. PNAS 1991.88:10357-10361.

How to cite this article: J.K. Chhetri, Q.-L. Xue, L. Ma et al. Intrinsic Capacity as a Determinant of Physical Resilience in Older Adults. J Nutr Health Aging. 2021;25(8):1006-1011; http://10.1007/s12603-021-1629-z 